\title{
Kinematics of Globular Clusters in M49 and M87
}

\author{
Terry Bridges \\ Anglo-Australian Observatory, P.O. Box 296, Epping, NSW, Australia
}

\begin{abstract}
We present recent multi-object spectroscopy of globular clusters in the Virgo gEs M49 and M87. In M49, we have a total of 144 confirmed clusters out to 8 arcmin radius $\left(\sim 6 \mathrm{R}_{\text {eff }}\right.$ or $\left.35 \mathrm{kpc}\right)$. We find that the blue (metal-poor) clusters have both a higher velocity dispersion and rotation than the red (metal-poor) clusters. For the metal-rich population we place an upper limit of $(\mathrm{v} / \sigma)_{\text {proj }}<0.34$ at $99 \%$ confidence. We calculate the velocity dispersion as a function of radius, and show that this is consistent with isotropic cluster orbits and the M49 mass distribution determined from X-ray data. For M87, we combine new CFHT data with previous data to obtain a total sample of 278 clusters out to 10 arcmin radius $(\sim 45 \mathrm{kpc})$. We find a similar global rotation for the metal-poor and metal-rich clusters of $160-170 \mathrm{~km} / \mathrm{sec}$. Beyond $\simeq 2 \mathrm{R}_{\text {eff }}(15 \mathrm{kpc})$, both the metal-poor and metal-rich clusters appear to rotate about the photometric minor axis. The combined cluster sample is consistent with isotropic orbits, but when considered separately, the metal-poor clusters show significant tangential bias of $\beta_{c l} \simeq-0.4$, while the metal-rich clusters show a radial bias with $\beta_{c l} \simeq+0.4$. In both galaxies, the metal-rich and metal-poor clusters share different kinematics, but there is no clear preference for any one formation scenario.
\end{abstract}

\section{Introduction}

There is still considerable controversy about how and when elliptical galaxies form. As survivors from the earliest epochs of galaxy formation, globular clusters (GCs) are incredibly useful probes of their host galaxies. Of great recent interest has been the finding of bimodality in the GC colour distributions in many elliptical galaxies. Several models have been put forward to explain this bimodality, including spiral-spiral mergers (Schweizer 1987; Ashman \& Zepf 1992), multiphase collapse (Forbes, Brodie, \& Grillmair 1997), and hierarchical accretion (Côté, Marzke, \& West 1998).

The kinematics and ages of metal-poor and metal-rich GCs around ellipticals will be key to distinguishing between these different scenarios, hence the importance of cluster spectroscopy. However, this spectroscopy is difficult, given the faintness of GCs in even the nearest ellipticals. It has only been with the advent of multi-object spectrographs on $4 \mathrm{~m}$ and $8-10 \mathrm{~m}$ telescopes that real progress has been made in this area. However, the sample of ellipticals with signficant numbers of GC spectra is still very small, limited to giant elliptical 
(gE) galaxies such as Centaurus A, NGC 1399, and M49 and M87 in Virgo. Here we present recent multi-slit spectroscopy of GCs in the latter two galaxies.

\section{M49 Globular Clusters}

With K. Ashman, M. Beasley, D. Geisler, D. Hanes, R. Sharples, and S. Zepf. See Sharples et al. (1998) and Zepf et al. (2000) for further details of the $M 49$ GC kinematics, and Beasley et al. (2000) regarding the $G C$ ages and metallicities.

\subsection{Sample Selection and Observations}

Object selection was based on Washington photometry of M49 cluster candidates from Geisler, Lee, \& Kim (1996). To improve the GC yield, we imposed magnitude and colour cuts: $19.5<\mathrm{V}<22.5,0.5<\mathrm{C}-\mathrm{T}_{1}<2.2$. Cluster spectra were obtained at the WHT with LDSS in 1994, and at the CFHT with MOS in 1998. Spectra covered the region from $3800-6000 \AA$ with a resolution of $3-6$ $\AA$, giving velocities good to $50-100 \mathrm{~km} / \mathrm{sec}$; exposure times were $3-4$ hours per mask. The combined cluster sample from the two runs (also including some GCs from Mould et al. 1990) is 144; of these, 93 are blue/metal-poor and 51 are $\mathrm{red} /$ metal-rich (dividing at $\mathrm{C}-\mathrm{T}_{1}=1.625$, or $[\mathrm{Fe} / \mathrm{H}] \sim-1.6$ ). We suffered only $15 \%$ contamination from non-clusters, and our overall completness was $65 \%$. Our sample extends out in radius to $\sim 8 \operatorname{arcmin}\left(\sim 6 \mathrm{R}_{\text {eff }}\right.$, or $\sim 35 \mathrm{kpc}$ for $\mathrm{D}=15 \mathrm{Mpc}$ ). Cluster velocities were obtained from cross-correlation with radial velocity stars.

\subsection{Results}

Kinematics Figure 1(a) shows the GC rotation and velocity dispersion radial profiles, for the total sample, and for the blue and red GCs separately. These profiles were obtained by smoothing with a Gaussian kernel with $\sigma=100^{\prime \prime}$. The blue GCs have a significantly larger velocity dispersion than the red GCs $(300-350 \mathrm{~km} / \mathrm{sec}$ compared to $\sim 200 \mathrm{~km} / \mathrm{sec})$, as already found by Sharples et al. (1998). Figure 1(a) shows that the M49 GCs have a slowly declining velocity dispersion profile, seen in both the full and blue/red samples. There is little evidence for rotation in the red GCs (but some evidence that the rotation increases outwards), while the rotation is roughly constant for the blue GCs. At all radii, the rotation is much smaller than the dispersion, and for the red GCs we can set an upper limit of $(\mathrm{v} / \sigma)_{\text {proj }}<0.34$ at $99 \%$ confidence; thus, rotation is not dynamically important for the M49 GCs.

$M / L$ Ratio and Orbital Anisotropy We use the velocity dispersion and luminosity density profiles, together with the Jeans equation, to estimate the M49 mass distribution; we assume isotropic orbits and spherical symmetry. Figure 1(b) shows the M49 mass vs. radius; the lines represent our cluster data, and the points with error bars are the masses inferred from ROSAT X-ray data (Irwin \& Sarazin 1996). There is reasonable agreement between the two mass determinations, suggesting that both are roughly correct, and that our assumption of isotropic cluster orbits isn't too far wrong. We find a total M49 mass in excess 

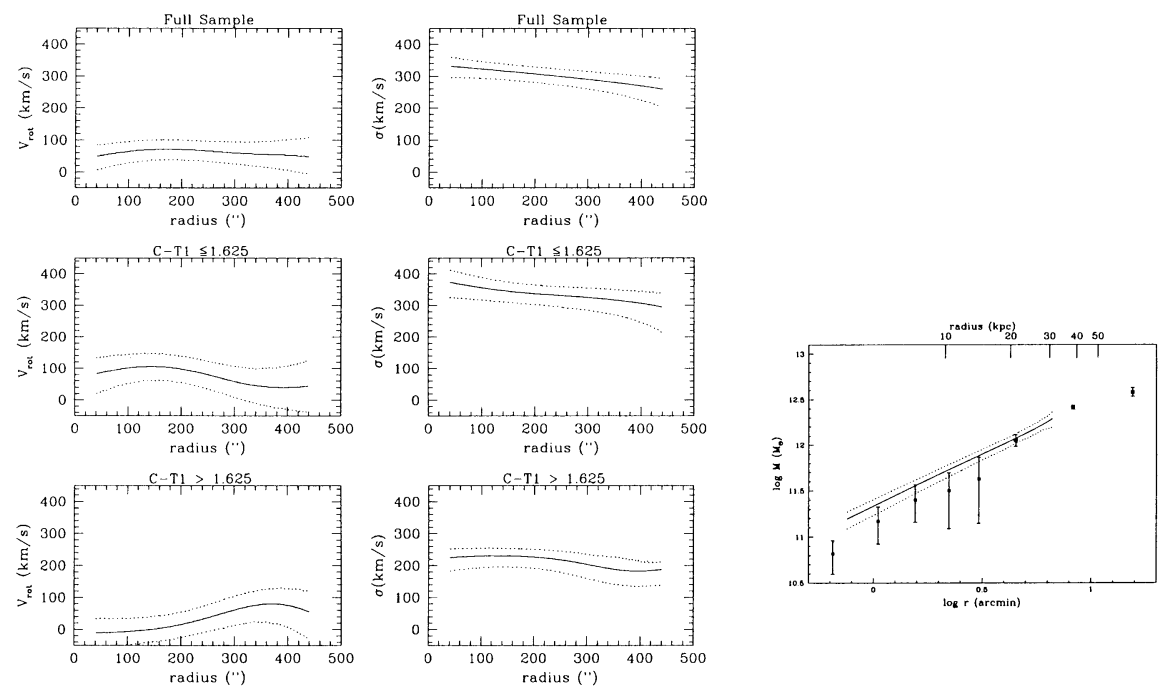

Figure 1. (a) (left): Smoothed rotation and velocity dispersion profiles for the M49 GCs. Top panels: combined sample; Middle panels: blue (metal-poor) GCs; Bottom panels: red (metal-rich) GCs. Dotted lines show the $1 \sigma$ bootstrapped uncertainties.

(b) (right): M49 mass vs. radius. Solid line: mass determined from the cluster radial velocities, assuming isotropic orbits; Dotted lines: $1 \sigma$ bootstrapped uncertainties; Points with error bars: masses from ROSAT X-ray data (Irwin \& Sarazin 1996).

of $10^{12} \mathrm{M}_{\odot}$, and a M/L ratio at least five times greater at $30 \mathrm{kpc}$ than at a few $\mathrm{kpc}$, confirming the existence of a substantial dark matter halo in M49.

\section{M87 Globular Clusters}

with P. Côté, D. Geisler, D. Hanes, G. Harris, J. Hesser, D. McLaughlin, and D. Merritt. See Hanes et al. (2001) and Côté et al. (2001) for further details.

\subsection{Observations and Final Sample}

Object selection was based on Washington $\mathrm{C}, \mathrm{T}_{1}$ imaging (Geisler, Lee, \& Kim 2001), with $0.8 \leq \mathrm{C}-\mathrm{T}_{1} \leq 2.35$. Spectra were obtained at the CFHT in 1996 with MOS, with a dispersion of $3.6 \AA /$ pixel. We used a filter centered at $5100 \AA$ with a bandwidth of $1200 \AA$ to allow us to place up to 100 slits per mask without spectral overlap. Galactic GCs were observed as radial velocity templates. We obtained velocities for 109 M87 GCs, 54 of these new measurements. By combining with previous work (most importantly, 205 velocities from Cohen \& Ryzhov 1997), we obtain a final sample of 278 bona-fide M87 GC velocities. Of 
these, 161 are classified as "blue" $\left(\mathrm{C}-\mathrm{T}_{1}<1.4\right)$ or metal-poor $([\mathrm{Fe} / \mathrm{H}]<-1.15)$ and 117 as "red" or metal-rich $\left(\mathrm{C}-\mathrm{T}_{1}>1.4,[\mathrm{Fe} / \mathrm{H}]>-1.15\right)$.

\subsection{Results}

Cluster Kinematics We investigate the global rotation and velocity dispersion of the M87 GCS by fitting our velocities with the function $v_{p}(\Theta)=v_{s y s}+$ $(\Omega R) \sin \left(\Theta-\Theta_{0}\right)$, where $\Theta_{0}$ is the GCS rotation axis, and $\Omega$ R is the rotation amplitude. When we average over all radii, we find similar rotation, dispersion, and position angles for the full sample, and for the blue and red clusters separately: $\Omega \mathrm{R} \sim 160-170 \mathrm{~km} / \mathrm{sec}$, a dispersion of $360-400 \mathrm{~km} / \mathrm{sec}$, and a position angle between $60-75^{\circ}$.

When we look at the kinematics as a function of radius, however, it starts to get interesting. Figure 2(a) shows the cluster rotation amplitude vs. radius for the total sample, and for the red and blue clusters separately, while Figure 2(b) shows the cluster best-fit position angle vs. radius. These (smoothed) curves were obtained by sliding a bin of radial width $\Delta \mathrm{R}=90^{\prime \prime}(\simeq 6.5 \mathrm{kpc})$ through the cluster data, and solving for the kinematics in each bin. From Figure 2(a), we see that the rotation is roughly constant with radius, but there is some evidence that the rotation increases at larger radius, especially for the blue GCs. Figure 2 (b) shows that, at large radius, the blue GCs rotate conventionally around the minor axis of $\mathrm{M} 87$, but for $\mathrm{R} \leq 16-18 \mathrm{kpc}$, they appear to rotate about the major axis! Similar behavior is seen in the red GCs, though not as pronounced. This flip in the rotation axis may be evidence for a past major merger.

Dynamical Models and Cluster Orbits Our dynamical approach is to assume a total M87 mass profile, as given by McLaughlin (1999). We plug this mass profile, the deprojected globular cluster surface density profile, and the velocity anisotropy $\beta_{c l}(r) \equiv 1-\sigma_{\theta}^{2} / \sigma_{r}^{2}$ into the Jeans equation and solve for the radial velocity dispersion $\sigma_{r}(\mathrm{r})$. A deprojection then gives the projected velocity dispersion profile $\sigma_{p}(\mathrm{r})$, which can then be compared with the observed profiles. Thus, we input various values of $\beta$ [specifically $\beta=-0.99$ (strong tangential bias), -0.4 (moderate tangential bias), 0 (isotropic), +0.4 (moderate radial bias), and 0.99 (strong radial bias)], and see which value fits the data best.

Figures $3(\mathrm{a}-\mathrm{c})$ show the results of this, for the combined sample, and for the metal-poor and metal-rich GCs separately. From Figure 3(a), the M87 GCS as $a$ whole has an almost perfectly isotropic velocity ellipsoid. However, the metalpoor GCs appear to have a modest tangential bias, with $\beta_{c l} \sim-0.4$ at small radii, while the metal-rich GCs appear to have a slight radial bias of roughly the same magnitude, $\beta_{c l} \sim+0.4$. Thus, the metal-poor and metal-rich GCs appear to have quite different orbits.

\section{Discussion: Comparison of M49 and M87}

What can we learn by comparing the M49 and M87 GCSs?

- In M49, there is little or no rotation in the red GCs, and $\sim 100 \mathrm{~km} / \mathrm{sec}$ rotation for the blue GCs. In M87, there is a similar rotation of $\sim 170$ $\mathrm{km} / \mathrm{sec}$ for both red and blue GCs. 

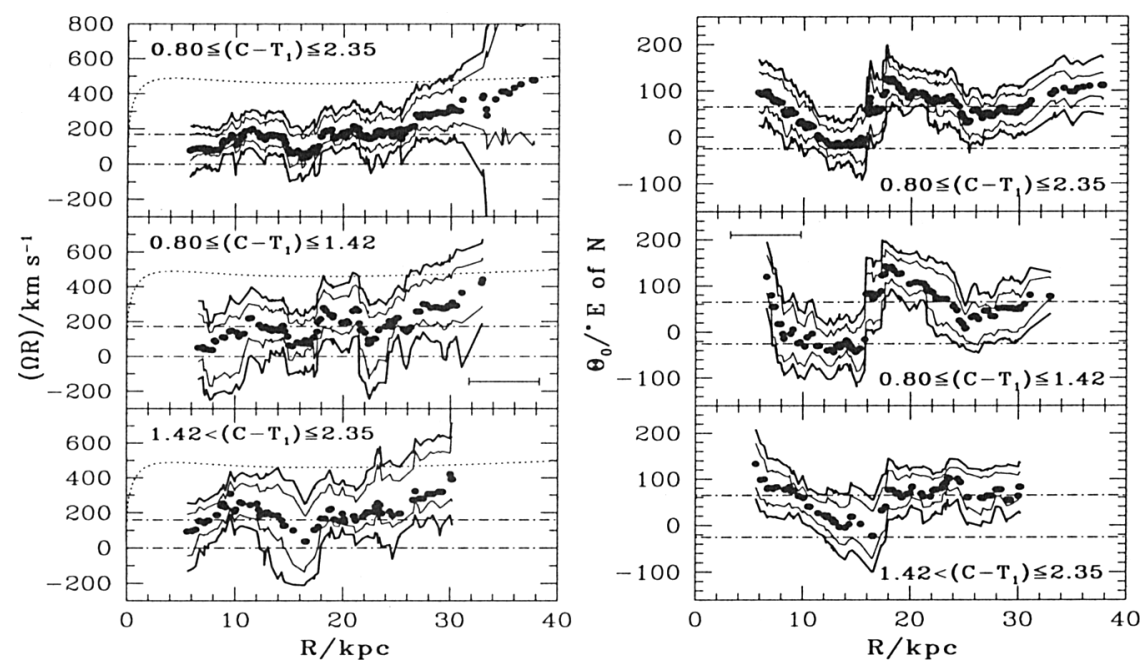

Figure 2. (a) (left): Projected rotation velocity vs galactocentric radius for the M87 GCS (filled circles), with $68 \%$ and $90 \%$ confidence bands (thin and thick solid lines). The smooth, dotted curves represent the circular velocity of the M87/Virgo potential from the model in McLaughlin 1999. Upper panel: combined sample; Middle panel: metal-poor GCs; Bottom panel: metal-rich GCs.

(b) (right): Same as Figure 2(a), but now plotting the projected azimuth of the rotation axis of the M87 GCS vs galactocentric radius. The lower and upper horizontal lines show the position angles of the M87 major and minor axes.

- In M49, there is little or no evidence that the rotation or velocity dispersion is rising with radius, whereas in M87 there is a suggestion that both the rotation and dispersion are increasing outwards. This is most likely because M87 is at the center of Virgo, and the M87 GCs are tracing the transition to the Virgo cluster potential. See Côté et al. (2001) for more discussion on this point.

- In M49, V/ $\sigma \sim 0.3 / 0.1$ for the blue/red GCs, while in M87 V/ $\sigma \sim 0.45$ for both subpopulations.

- The combined samples of GCs in M49 and M87 are both consistent with isotropy. In M87, the blue GCs appear to have a tangential bias, while the red GCs have a radial bias.

Thus, in both galaxies, the metal-poor and metal-rich GCs have different kinematics (rotation, velocity dispersion, and orbital anisotropy), but these differences are not consistent between the two galaxies. It is difficult to think of a single formation model that can explain the properties (kinematic and other) of both GCSs. There is obviously a huge need for further kinematical samples in a wider range of galaxies. Luckily, we will soon have fabulous new multi-slit 

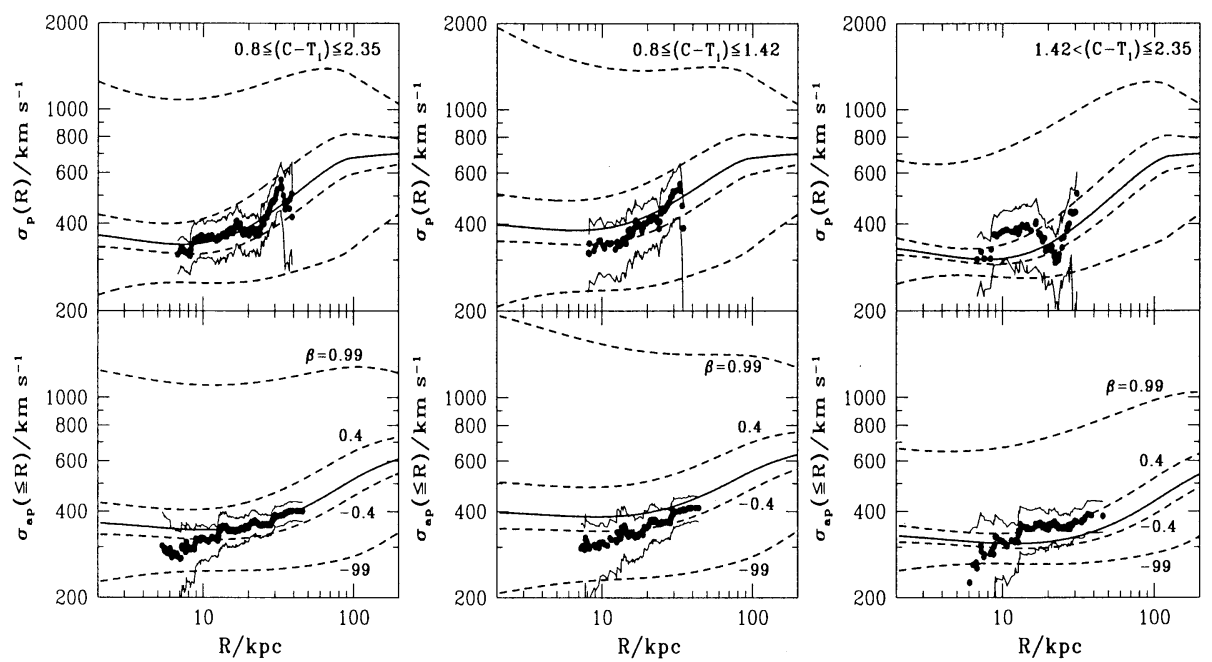

Figure 3. (a) (left): Upper Panel: Velocity dispersion profile for the combined sample of M87 GCs. Points: smoothed data (the thin solid curves are $90 \%$ confidence intervals); Thick solid line: predicted velocity dispersion profiles for isotropic orbits $(\beta=0)$; Dashed lines: $\beta=0.99$, $0.4,-0.4$, and -0.99 , from top to bottom. Lower Panel: similar to upper panel, except now for the aperture velocity dispersion profile.

(b) (middle): Same as Fig 3(a), except for the metal-poor GCs.

(c) (right): Same as Fig 3(a), except for the metal-rich GCs.

spectrographs on 8m telescopes: GMOS on Gemini and VIMOS on the VLT. The next few years will be very exciting.

Acknowledgments. I'd like to thank my collaborators for allowing me to discuss our work at this conference, and for the pleasure of working with them. I'd also like to thank the organizers, particularly Doug and Eva, for arranging such a fantastic conference, and for financial support. It was a wonderful week.

\section{References}

Ashman, K.M., \& Zepf, S.E. 1992, ApJ, 384, 50

Beasley, M.A., Sharples, R.M., Bridges, T.J., Hanes, D.A., Zepf, S.E., Ashman, K.M., \& Geisler, D. 2000, MNRAS, 318, 1249

Cohen, J.G., \& Ryzhov, A. 1997, ApJ, 486, 230

Côté, P., Marzke, R.O., \& West, M.J. 1998, 501, 554

Côté, P., McLaughlin, D.E., Hanes, D.A., Bridges, T.J., Geisler, D., Merritt, D., Hesser, J.E., Harris, G.L.H., \& Lee, M.G. 2001, ApJ, in press (astroph 0106005)

Forbes, D.A., Brodie, J.P., \& Grillmair, C.J. 1997, AJ, 113, 1652

Geisler, D., Lee, M.G., \& Kim, E. 1996, AJ, 111, 1529 
Geisler, D., Lee, M.G., \& Kim, E. 2001, in preparation

Hanes, D.A., Côté, P., Bridges, T.J., McLaughlin, D.E., Geisler, D., Harris, G.L.H., Hesser, J.E., \& Lee, M.G. 2001, ApJ, in press (astroph 0106004)

Irwin, J.A., \& Sarazin, C.L. 1996, ApJ, 471, 683

McLaughlin, D.E. 1999, ApJ, 512, L9

Mould, J.R., Oke, J.B., de Zeeuw, P.T., \& Nemec, J. 1990, AJ, 99, 1823

Schweizer, F. 1987, in Nearly Normal Galaxies, (New York: Springer-Verlag), 18

Sharples, R.M., Zepf, S.E., Bridges, T.J., Hanes, D.A., Carter, D., Ashman, K.M., \& Geisler, D. 1998, AJ, 115, 2337

Zepf, S.E., Beasley, M.A., Bridges, T.J., Hanes, D.A., Sharples, R.M., Ashman, K.M., \& Geisler, D. 2000, AJ, 120, 2928

\section{Discussion}

A. Kundu: 1). How is the minor axis defined in M87? 2). Does the rotation axis in the inner region match up with the flattening of the GCLF azimuthal distribution in Kundu et al? 3). Is the break in the rotation axis consistent with the break in GC properties found by Harris et al?

T. Bridges: 1). The minor axis is taken as a global average. We take the minor axis position angle to be $\Theta_{\text {minor }}=65^{\circ}$ from Carter \& Dixon (1978, AJ, 83, 6). 2 ). I don't know 3). Yes, both occur at $R=15-20 \mathrm{kpc}$. This is probably not an accident. 